\title{
Analyticity Properties of Eigenfunctions and Scattering Matrix *
}

\author{
Erik Balslev
}

University of Aarhus, Denmark and Institute for Advanced Study, Princeton, NJ 08540, USA

\begin{abstract}
For potentials $V=V(x)=O\left(|x|^{-2-\varepsilon}\right)$ for $|x| \rightarrow \infty, x \in \mathbb{R}^{3}$, we prove that if the $S$-matrix of $(-\Delta,-\Delta+V)$ has an analytic extension $\widetilde{S}(z)$ to a region $\mathcal{O}$ in the lower half-plane, then the family of generalized eigenfunctions of $-\Delta+V$ has an analytic extension $\tilde{\phi}(k, \omega, x)$ to $\mathcal{O}$ such that $|\tilde{\phi}(k, \omega, x)|<C e^{b|x|}$ for $|\operatorname{Im} k|<b$. Consequently, the resolvent $\left(-\Delta+V-z^{2}\right)^{-1}$ has an analytic continuation from $\mathbb{C}^{+}$to $\{k \in \mathcal{O}|| \operatorname{Im} k \mid<b\}$ as an operator $\widetilde{R}(z)$ from $\mathscr{H}_{b}=\{f$ $\left.=e^{-b|x|} g \mid g \in L_{2}\left(\mathbb{R}^{3}\right)\right\}$ to $\mathscr{H}_{-b}$. Based on this, we define for potentials $W=o\left(e^{-2 b|x|}\right)$ resonances of $(-\Delta+V,-\Delta+V+W)$ as poles of $(1+W \widetilde{R}(z))^{-1}$ and identify these resonances with poles of the analytically continued $S$-matrix of $(-\Delta+V,-\Delta+V+W)$.
\end{abstract}

\section{Introduction}

Analytic continuation of the scattering matrix of a two-body Schrödinger operator $-\Delta+V$ has been established for various classes of the potential $V$, including exponentially decaying [3] and dilation-analytic, short-range [4] potentials.

Two methods were developed to obtain a unified approach to these two classes of potentials, one [5] based on local spectral deformation techniques in momentum space, the other [9] based on an analytic family of deformations of the underlying momentum-space. These methods cover potentials of the form $V+W$, where $V=O\left(r^{-2-\varepsilon}\right)$ is radial, dilation-analytic and $W$ is exponentially decaying.

For radial potentials a different method was introduced [6]. The basic idea was that if the resolvent $\left(-\Delta+V-k^{2}\right)^{-1}$ can be shown to have an analytic continuation to a domain $\mathcal{O}$ in the lower half-plane as an operator from a space of exponentially decaying functions to its dual, then $-\Delta+V$ can play the role of $-\Delta$ as background for an exponentially decaying perturbation $W$, using analytic

* The author would like to thank the Institute for Advanced Study for its hospitality and the National Science Foundation for financial support under Grant No. DMS-8610730(1) 
Fredholm theory. In particular it was shown that if the scattering matrix of $(-\Delta$, $-\Delta+V$ ) has an analytic extension to $\mathcal{O}$, then the resolvent has such an analytic continuation.

In the present paper we extend these results to non-radial potentials. In Sect. 1 we establish the existence of an analytic extension to the upper half-plane of the generalized eigenfunctions $\psi_{+}(k, \omega, x)$ defined in a standard way for $k$ real. The construction utilizes methods of [1], extended to operators of the type $e^{-i k \omega \cdot x} \Delta e^{i k \omega \cdot x}$. The proof requires that $V(x)=O\left(|x|^{-2-\varepsilon}\right)$, but it is possible to extend these results to potentials $V(x)=O\left(|x|^{-1-\varepsilon}\right)$ (cf. $[10,11]$ ) and perhaps even, with modifications, to long potentials. A different method of obtaining the analytic extension of the eigenfunctions to the upper half-plane via the Green's function is indicated in [2].

In Sect. 2 we prove that $\psi(k, \omega, x)$ can be continued analytically in $k$ to a domain $\mathcal{O}$ in the lower half-plane, provided the $S$-matrix $S_{1}(k)$ can be extended in this way (Theorem 2.2). We utilize the abstract stationary scattering theory as developed in [8]. From this we obtain analytic continuation of the resolvent $\left(-\Delta+V-k^{2}\right)^{-1}$, acting as an operator from an exponentially weighted $L_{2}$-space into its dual (Theorem 2.4). This allows for addition of an exponentially decaying potential $W$ to $V$. As a consequence we obtain as our main result (Theorems 2.5 and 2.7) the meromorphic extension of the $S$-matrices $S_{12}(k)$ of $(-\Delta+V,-\Delta$ $+V+W)$ and $S_{2}(k)$ of $(-\Delta,-\Delta+V+W)$. This provides a basis for the further study of resonances and resonance functions arising from a very short range potential $W$ acting on the background of a short range smooth potential $V$. This will be taken up in [11].

\section{Generalized Eigenfunctions}

In this section we define the generalized eigenfunctions $\Psi(k, \omega, x)$ for $k \in \overline{\mathbb{C}^{+}} \backslash\{0\}$, $\omega \in S^{2}$ and $x \in \mathbb{R}^{3}$. We establish the analyticity properties of $\Psi(k, \omega, x)$ as functions of $k \in \overline{\mathbb{C}^{+}} \backslash\{0\}$ and their exponential growth as functions of $x \in \mathbb{R}^{3}$.

The free Hamiltonian $H_{0}$ is the selfadjoint operator defined by $H_{0} f=-\Delta f$ with domain $\mathscr{D}_{\mathrm{H}_{0}}=\mathrm{H}_{2}\left(\mathbb{R}^{3}\right)$. The potential $V$ is assumed to be multiplication by a real-valued, measurable function $v$ on $\mathbb{R}^{3}$ satisfying for some $\varepsilon>0, R_{0}>0$,

(i) $v \in L_{2}^{\text {loc }}\left(\mathbb{R}^{3}\right)$,

(ii) $|v(x)|<C|x|^{-2-\varepsilon}$ for $|x|>R_{0}$.

$V$ is $H_{0}$-compact and hence $H_{0}-\varepsilon$-bounded. Thus, the Hamiltonian $H_{1}=H_{0}+V$ is selfadjoint on $\mathscr{D}_{H_{1}}=\mathscr{D}_{H_{0}}$, and $\sigma_{e}\left(H_{1}\right)=\overline{\mathbb{R}^{+}}$.

Two families of generalized eigenfunctions $\Psi_{ \pm}(k, \omega, x)$ are formally defined [1] for $k>0, \omega \in S^{2}, x \in \mathbb{R}^{3}$ by

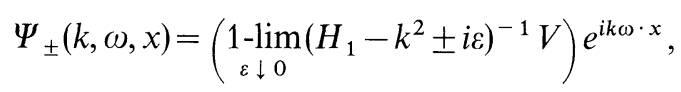

where the limit is taken in a suitable topology. 
For the purpose of studying analyticity properties it is useful to introduce the family of eigenfunctions $\Psi(k, \omega, x)$ defined formally for $k \in \overline{\mathbb{C}^{+}} \backslash\{0\}$ by

$$
\Psi(k, \omega, x)=\lim _{\varepsilon \downarrow 0}\left(1-R_{1}((k+i \varepsilon)) V\right) e^{i k \omega \cdot x},
$$

where $R_{1}(k+i \varepsilon)=\left(H_{1}-(k+i \varepsilon)^{2}\right)^{-1}$.

The connection between $\Psi_{ \pm}(k, \omega, x)$ and $\Psi(k, \omega, x)$ is given for $k>0$ by

$$
\Psi_{+}(k, \omega, x)=\Psi(-k,-\omega, x), \quad \Psi_{-}(k, \omega, x)=\Psi(k, \omega, x) .
$$

We shall prove that the functions $\Psi(k, \omega, x)$ exist and behave asymptotically as $e^{i k \omega \cdot x}$. For this purpose we study the functions $\Phi(k, \omega, x)$ formally defined by

$$
\begin{aligned}
\Phi(k, \omega, x) & =1-e^{i k \omega \cdot x} \Psi(k, \omega, x)=e^{-i k \omega \cdot x} R_{1+}(k) V e^{i k \omega \cdot x} \\
& =\left(1-e^{-i k \omega \cdot x} R_{0+}(k) e^{i k \omega \cdot x} V\right)^{-1} e^{-i k \omega \cdot x} R_{0+}(k) e^{-i k \omega \cdot x} V,
\end{aligned}
$$

where in a suitable topology

$$
R_{i+}(k)=\lim _{\varepsilon \downarrow 0} R_{i}((k+i \varepsilon)), \quad i=0,1 .
$$

The basic operator is $R_{0+}(k, \omega)$ defined for $k \in \overline{\mathbb{C}^{+}}, \omega \in S^{2}$, by

$$
\begin{aligned}
R_{0+}(k, \omega) & =e^{-i k \omega \cdot x} R_{0+}(k) e^{i k \omega \cdot x}=\left(e^{-i k \omega \cdot x}\left(H_{0}-k^{2}\right) e^{i k \omega \cdot x}\right)_{+}^{-1} \\
& =\left(T_{\omega} e^{-i k x_{1}} T_{\omega}^{-1}\left(H_{0}-k^{2}\right) T_{\omega} e^{i k x_{1}} T_{\omega}^{-1}\right)_{+}^{-1} \\
& =T_{\omega}\left(e^{-i k x_{1}}\left(H_{0}-k^{2}\right) e^{i k x_{1}}\right)_{+}^{-1} T_{\omega}^{-1}=T_{\omega}\left(H_{0}-2 i k \frac{\partial}{\partial x_{1}}\right)_{+}^{-1} T_{\omega}^{-1},
\end{aligned}
$$

where $T_{\omega}$ is the operator defined by

$$
\left(T_{\omega} f\right)(x)=f\left(t_{\omega}^{-1} x\right),
$$

and $t_{\omega}$ is any rotation which sends $(1,0,0)$ into $\omega$.

Setting $k=\alpha+i \beta$, we have

$$
H_{0}-2 i k \frac{\partial}{\partial x_{1}}=e^{-i \alpha x_{1}} e^{\beta x_{1}} H_{0} e^{-\beta x_{1}} e^{i \alpha x_{1}}-k^{2}=e^{-i \alpha x_{1}}\left(H_{0}(i \beta)-k^{2}\right) e^{i \alpha x_{1}},
$$

where

$$
H_{0}(i \beta)=e^{\beta x_{1}} H_{0} e^{-\beta x_{1}}=H_{0}+2 \beta \frac{\partial}{\partial x_{1}}-\beta^{2}
$$

(see also Appendix 2).

The operator $H_{0}(i \beta)$ has as its spectrum the parabolic region $\mathscr{P}_{\beta}=\left\{z^{2}|| \operatorname{Im} z \mid \leqq \beta\right\}$. The point $k^{2}$ belongs to the boundary of $\mathscr{P}_{\beta}$, and for $\beta \geqq 0$ fixed, $\varepsilon>0,(k+i \varepsilon)^{2} \in \mathbb{C} \backslash \mathscr{P}_{\beta}$,

$$
R_{0}(\beta, k+i \varepsilon)=\left(H_{0}(i \beta)-(k+i \varepsilon)^{2}\right)^{-1} \in \mathscr{B}(\mathscr{H}),
$$

and

$$
\left(H_{0}-2 i k \frac{\partial}{\partial x_{1}}\right)_{+}^{-1}=e^{i \alpha x_{1}} R_{0+}(\beta, k) e^{-i \alpha x_{1}}
$$


where

$$
R_{0+}(\beta, k)=\lim _{\varepsilon \downarrow 0} R_{0}(\beta, k+i \varepsilon) .
$$

The rest of this section is devoted to a rigorous derivation of the above formalism.

Definition. For $s \in \mathbb{R}$ we define the following spaces:

$$
\begin{gathered}
L_{2, s}^{1}=L_{2, s}^{1}\left(\mathbb{R}^{3}\right)=\left\{\left.f\left|\|f\|_{L_{2, s}^{1}}^{2}=\int_{\mathbb{R}^{3}}\right| f(x)\right|^{2}\left(1+x_{1}^{2}\right)^{s} d x<\infty\right\}, \\
L_{2, s}=L_{2, s}\left(\mathbb{R}^{3}\right)=\left\{\left.f\left|\|f\|_{L_{2, s}}^{2}=\int_{\mathbb{R}^{3}}\right| f(x)\right|^{2}\left(1+|x|^{2}\right)^{s} d x<\infty\right\}, \\
H_{2, s}=H_{2, s}\left(\mathbb{R}^{3}\right)=\left\{f \mid D^{\alpha} f \in L_{2, s} \text { for }|\alpha| \leqq 2\right\},
\end{gathered}
$$

and

$$
\|f\|_{H_{2, s}}^{2}=\sum_{|\alpha| \leqq 2}\left\|D^{\alpha} f\right\|_{L_{2, s}}^{2}
$$

For $b \in \mathbb{R}$,

$$
\begin{aligned}
& \mathscr{H}^{b}=\left\{\left.f\left|\|f\|_{\mathscr{H}^{b}}^{2}=\int_{\mathbb{R}^{3}} e^{2 b|x|}\right| f(x)\right|^{2} d x<\infty\right\}, \\
& \mathscr{H}_{2}^{b}=\left\{f \mid D^{\alpha} f \in \mathscr{H}^{b} \text { for }|\alpha| \leqq 2\right\},
\end{aligned}
$$

and

$$
\|f\|_{\mathscr{H}_{2}^{b}}^{2}=\sum_{|\alpha| \leqq 2}\left\|D^{\alpha} f\right\|_{\mathscr{H}^{b}}^{2}
$$

We set

$$
\mathscr{H}=L_{2}\left(\mathbb{R}^{3}\right), \quad \mathfrak{H}=L^{2}\left(S^{2}\right) .
$$

$\mathscr{H}_{\mathfrak{H}}^{b}$ and $\mathscr{H}_{2, \mathfrak{H}}^{b}$ are defined as $\mathscr{H}^{b}$ and $\mathscr{H}_{2}^{b}$ with $f$ replaced by an $\mathfrak{H}$-valued function on $\mathbb{R}^{3}$ and $|f(x)|$ replaced by $\|f(x)\|_{\mathfrak{S}}$. by

We consider for $\beta \geqq 0$ the closed operator $H_{0}(i \beta)$ with domain $H_{2}\left(\mathbb{R}^{3}\right)$, defined

$$
H_{0}(i \beta)=-\Delta+2 \beta \frac{\partial}{\partial x_{1}}-\beta^{2},
$$

whose Fourier transform is multiplication by the polynomial

$$
Q(\beta)=\xi^{2}+2 i \beta \xi_{1}-\beta^{2} .
$$

Lemma 1.1. For given $s>\frac{1}{2}, \beta_{0}>0, K$ a compact subset of $\mathbb{C} \backslash\{0\}$, there exists a constant $C$ such that for $0 \leqq \beta \leqq \beta_{0}, z \in K, u \in H_{2}$

$$
\|u\|_{L_{2,-s}^{1}} \leqq C\left\|\left(H_{0}(i \beta)-z\right) u\right\|_{L 1, s} .
$$

Proof. The lemma is proved in the same way as [1, Lemma A.1]. Note that the set of critical points $\Lambda_{c}(Q(\beta))$ of $Q(\beta)$ is given by

$$
\Lambda_{c}(Q(\beta))=\left\{Q(\beta) \xi \mid \nabla_{\xi} Q(\beta)=0\right\}=\left\{\begin{array}{lll}
\emptyset & \text { if } & \beta \neq 0 \\
\{0\} & \text { if } & \beta=0
\end{array} .\right.
$$


It is easy to check that each step of the proof given in [1] is valid with $\left(1+|x|^{2}\right)^{s}$ replaced by $\left(1+x_{1}^{2}\right)^{s}$ and that $C$ can be chosen independent of $\beta, 0 \leqq \beta \leqq \beta_{0}$.

For the construction of generalized eigenfunctions we shall make use of the boundary values $R_{0+}(\beta, k), k=\alpha+i \beta$. The existence of these boundary values is proved through a series of lemmas. In this section $K$ denotes a compact subset of $\overline{\mathbb{C}^{+}} \backslash\{0\}$.

Lemma 1.2. Let $g \in L_{2, s}^{1}, s>\frac{1}{2}$. For $k \in \overline{\mathbb{C}^{+}} \backslash\{0\}$ the following weak limits exist in $H_{2,-s}^{1}$, uniformly for $k \in K$,

$$
R_{0+}(\beta, k) g=w-\lim _{\varepsilon \downarrow 0} R_{0}(\beta, k+i \varepsilon) g .
$$

Proof. For $f$ and $g$ with compact support

$$
\left(f, R_{0}(\beta, k+i \varepsilon) g\right)=\left(e^{\beta x_{1}} f, R_{0}(k+i \varepsilon) e^{-\beta x_{1}} g\right) \underset{\varepsilon \downarrow 0}{\longrightarrow}\left(e^{\beta x_{1}} f, R_{0}(k) e^{-\beta x_{1}} g\right),
$$

uniformly for $k \in K \subset \mathbb{C}^{+}$. By Lemma A1 this also holds for $K \subset \overline{\mathbb{C}^{+}} \backslash\{0\}$. Then, by Lemma 1.1, the same holds for $f, g \in L_{2, s}^{1}$.

Thus, for $g \in L_{2, s}^{1}$ there exists

$$
w-\lim _{\varepsilon \downarrow 0} R_{0}(\beta, k+i \varepsilon) g \quad \text { in } \quad L_{2,-s}^{1} .
$$

By Lemma 1.1, $\left\{R_{0}(\beta, k+i \varepsilon) g\right\}$ is also bounded in $H_{2,-s}^{1}$ for $\varepsilon>0$, uniformly for $k \in K$. Hence, by the weak compactness of the unit ball in $H_{2,-s}^{1}$, the weak limit is attained also in $H_{2,-s}^{1}$, uniformly for $k \in K$.

Theorem 1.3. $R_{0+}(\beta, k)=\lim _{\varepsilon \downarrow 0} R_{0}(\beta, k+i \varepsilon)$ in the uniform operator topology of $\mathscr{B}\left(L_{2, s}^{1}, H_{2,-s}\right)$, uniformly for $k \in K$.

Moreover, the $\mathscr{B}\left(L_{2, s}^{1}, H_{2,-s}\right)$-valued function of $k=\alpha+i \beta$

$$
e^{-i \alpha x_{1}} R_{0+}(\beta, k) e^{i \alpha x_{1}}
$$

is analytic in $\mathbb{C}^{+}$and continuous in $\overline{\mathbb{C}^{+}} \backslash\{0\}$.

Proof. By Lemma 1.2, $R_{0+}(\beta, k):=w-\lim R_{0}(\beta, k+i \varepsilon)$ is well-defined as an operator in $\mathscr{B}\left(L_{2, s}^{1}, H_{2,-s}\right)$. Note that for $u \in H_{2,-s}$ the norm $\|u\|_{2,-s}$ is equivalent to

$$
\|u\|=\|u\|_{-s}+\left\|\left(-\Delta+2 \beta \frac{\partial}{\partial x_{1}}\right) u\right\|_{-s}
$$

uniformly for $0 \leqq \beta \leqq \beta_{0}$, where we fix $\beta_{0}>0$ such that $K \subset\left\{k \mid 0 \leqq \operatorname{Im} k \leqq \beta_{0}\right\}$. Thus it suffices to show that in the uniform operator topology of $\mathscr{B}\left(L_{2, s}^{1}, L_{2,-s}\right)$,

$$
R_{0}(\beta, k+i \varepsilon) \underset{\varepsilon \downarrow 0}{\longrightarrow} R_{0+}(\beta, k)
$$

and

$$
\left(-\Delta+2 \beta \frac{\partial}{\partial x_{1}}\right) R_{0}(\beta, k+i \varepsilon) \underset{\varepsilon \downarrow 0}{\longrightarrow}\left(-\Delta+2 \beta \frac{\partial}{\partial x_{1}}\right) R_{0+}(\beta, k),
$$

uniformly for $k \in K$. 
Since

$$
\left(-\Delta+2 \beta \frac{\partial}{\partial x_{1}}\right) R_{0}(\beta, k+i \varepsilon)=1+(k+i \varepsilon)^{2} R_{0}(\beta, k+i \varepsilon),
$$

it follows that it suffices to show that

$$
R_{0}(\beta, k+i \varepsilon) \underset{\varepsilon \downarrow 0}{\longrightarrow} R_{0+}(\beta, k)
$$

in the uniform operator topology of $\mathscr{B}\left(L_{2, s}^{1}, L_{2,-s}\right)$, uniformly for $k \in K$. We now proceed to show this.

First of all, the weak limit as $\varepsilon \downarrow 0$ is indeed a strong limit, which can be seen as follows. Let $g \in L_{2, s^{*}}^{1}$ Then $g \in L_{2, s}^{1}$, for $\frac{1}{2}<s^{\prime}<s$. By Lemma 1.2,

$$
R_{0}(\beta, k+i \varepsilon) g \underset{\varepsilon \downarrow 0}{\longrightarrow} R_{0+}(\beta, k) g,
$$

weakly in $H_{2,-s^{\prime}}^{1}$, uniformly for $k \in K$. Since $H_{2}^{1}, s^{\prime}$ is compactly embedded in $L_{2,-s}$, we conclude that

$$
R_{0}(\beta, k+i \varepsilon) g \underset{\varepsilon \downarrow 0}{\longrightarrow} R_{0+}(\beta, k) g,
$$

strongly in $L_{2,-s}$, uniformly for $k \in K$.

It remains to prove convergence in the uniform operator topology. This is proved along the same lines as the proof of the corresponding step in the proof of [1, Theorem 4.1], replacing $L_{2, s}$ by $L_{2, s}^{1}$ and $H_{2,-s^{\prime}}$ by $H_{2,-s}^{1}$ and using the compactness of the embedding of $H_{2,-s^{\prime}}^{1}$ in $L_{2,-s}$ for $\frac{1}{2}<s^{\prime}<s$. This concludes the proof of the first part of the theorem.

For $\varepsilon>0$ the $\mathscr{B}\left(L_{2, s}^{1}, H_{2,-s}\right)$-valued function $e^{-i \alpha x_{1}} R_{0}(\beta, k+i \varepsilon) e^{i \alpha x_{1}}$ is analytic in $k \in \mathbb{C}^{+}$and continuous in $k \in \overline{\mathbb{C}^{+}} \backslash\{0\}$.

It then follows from the first part of the theorem that $e^{-i \alpha x_{1}} R_{0+}(\beta, k) e^{i \alpha x_{1}}$ is analytic in $\mathbb{C}^{+}$and continuous in $\overline{\mathbb{C}^{+}} \backslash\{0\}$ as a function of $k$ with values in $\mathscr{B}\left(L_{2, s}^{1}, H_{2,-s}\right)$.

The theorem is proved.

Theorem 1.4. The following limits exist in the uniform operator topology of $\mathscr{B}\left(L_{2, s}^{1}, H_{2,-s}\right)$ for $s>\frac{1}{2}$, uniformly for $k$ in compact subsets of $\overline{\mathbb{C}^{+}} \backslash \Sigma$, where

$$
\begin{aligned}
\Sigma & =\left\{i \beta \mid-\beta^{2} \in \sigma_{d}(H)\right\} \cup\{0\} \cup\left\{\alpha \mid \alpha^{2} \in \sigma_{p}(H)\right\}, \\
R_{1+}(\beta, k) & =\lim _{\varepsilon \downarrow 0} R_{1}(\beta, k+i \varepsilon)=\left(1+R_{0+}(\beta, k) V\right)^{-1} R_{0+}(\beta, k) .
\end{aligned}
$$

Moreover, the $\mathscr{B}\left(L_{2, s}^{1}, H_{2,-s}\right)$-valued function $e^{-i \alpha x_{1}} R_{1+}(\beta, k) e^{i \alpha x_{1}}$ is meromorphic in $\mathbb{C}^{+}$with poles at $\left\{i \beta \mid-\beta^{2} \in \sigma_{d}(H)\right\}$ and continuous in $\mathbb{C}^{+} \backslash \Sigma$.

Proof. By the $2^{\text {nd }}$ resolvent equation,

$$
R_{1}(\beta, k+i \varepsilon)=\left(1+R_{0}(\beta, k+i \varepsilon) V\right)^{-1} R_{0}(\beta, k+i \varepsilon) .
$$

Since $V \in \mathscr{C}\left(H_{2,-s}, L_{2, s}\right)$, by Theorem 1.3,

$$
e^{-i \alpha x_{1}} R_{0}(\beta, k+i \varepsilon) V e^{i \alpha x_{1}} \underset{\varepsilon \downarrow 0}{\longrightarrow} e^{-i \alpha x_{1}} R_{0+}(\beta, k) e^{i \alpha x_{1}}:=Q_{+}(k)
$$


in the uniform operator topology of $\mathscr{B}\left(H_{2,-s}\right)$, uniformly for $k \in K$, and $Q_{+}(k)$ is an analytic, $\mathscr{C}\left(H_{2,-s}\right)$-valued function of $k \in \mathbb{C}^{+}$and continuous in $\overline{\mathbb{C}^{+}} \backslash\{0\}$. By Lemma A2, $4,1+Q_{+}(k)$ is invertible for $k \in \overline{\mathbb{C}^{+}} \backslash \Sigma$.

It follows from the analytic Fredholm theorem that $\left(1+Q_{+}(k)\right)^{-1}$ is a meromorphic, $\mathscr{B}\left(H_{2,-s}\right)$-valued function in $\mathbb{C}^{+}$with poles at $\Sigma_{i}=\left\{i \beta \mid-\beta^{2}\right.$

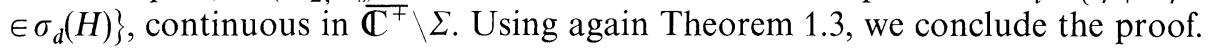

Theorem 1.5. Set for $k \in \overline{\mathbb{C}^{+}} \backslash \Sigma$,

$$
\Phi(k, \omega, x)=e^{-i k \omega \cdot x} R_{1+}(k) V e^{i k \omega \cdot x} .
$$

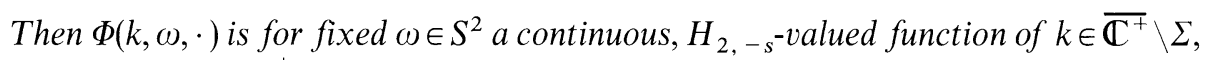
meromorphic in $\mathbb{C}^{+}$with poles at most at $\Sigma_{i}$.

For fixed $x \in \mathbb{R}^{3}, \omega \in S^{2}, \Phi(k, \omega, x)$ is continuous in $\overline{\mathbb{C}^{+}} \backslash \Sigma$ and meromorphic in $\mathbb{C}^{+}$with poles at most at $\Sigma_{i}$.

Proof. Since $V \in L_{2, s}^{1}$ and

$$
\Phi(k, \omega, x)=T_{\omega} e^{-i \alpha x_{1}} R_{1+}(\beta, k) e^{i \alpha x_{1}} T_{\omega}^{-1} V,
$$

the first part of the theorem is an immediate consequence of Theorem 1.4.

It follows that for fixed $\omega \in S^{2}, f \in C_{0}\left(\mathbb{R}^{3}\right)$, the function

$$
\int_{\mathbb{R}^{3}} \Phi(k, \omega, x) f(x) d x
$$

is continuous in $\overline{\mathbb{C}^{+}} \backslash \Sigma$ and meromorphic in $\mathbb{C}^{+}$with poles at most at $\Sigma_{i}$. Note that $\Phi(k, \omega, \cdot) \in H_{2,-s}$ implies that $\Phi(k, \omega, \cdot) \in C\left(\mathbb{R}^{3}\right)$. Let $\Gamma \subset \mathbb{C}^{+}$be a closed curve with $\Gamma \cap \Sigma_{i}=\emptyset$ and containing no points of $\Sigma_{i}$ in its interior. Then, by Fubini's theorem

$$
\int_{\mathbb{R}^{3}}\left\{\int_{\Gamma} \Phi(k, \omega, x) d k\right\} f(x) d x=\iint_{\Gamma}\left\{\int_{\mathbb{R}^{3}} \Phi(k, \omega, x) f(x) d x\right\} d k=0 .
$$

This implies that $\int_{\Gamma} \Phi(k, \omega, x) d k=0$ for fixed $\omega \in S^{2}, x \in \mathbb{R}^{3}$, and hence $\Phi(k, \omega, x)$ is analytic in $\mathbb{C}^{+} \backslash \Sigma_{i}$. It follows from a similar argument with $\Gamma$ containing a point of $\Sigma_{i}$ that such points are at most poles of $\Phi(k, \omega, x)$.

The continuity of $\Phi(k, \omega, x)$ is proved as follows. For fixed $\omega \in S^{2}$, the function $\left(1+|x|^{2}\right)^{-s / 2} \Phi(k, \omega, \cdot)$ is continuous in $k \in \overline{\mathbb{C}^{+}} \backslash \Sigma$ with values in $H_{2}$. Since $H_{2}$ is continuously embedded in $C\left(\mathbb{R}^{3}\right)$ (with the sup-norm), $\left(1+|x|^{2}\right)^{-s / 2} \Phi(k, \omega, \cdot)$ is continuous in $k \in \overline{\mathbb{C}^{+}} \backslash \Sigma$ with values in $C\left(\mathbb{R}^{3}\right)$. Hence for every $x \in \mathbb{R}^{3}, \omega \in S^{2}$, the function $\Phi(k, \omega, x)$ is continuous in $k \in \overline{\mathbb{C}}^{+} \backslash \Sigma$.

Definition 1.6. For $k \in \overline{\mathbb{C}^{+}} \backslash \Sigma, \omega \in S^{2}, x \in \mathbb{R}^{3}$,

$$
\Psi(k, \omega, x)=e^{i k \omega \cdot x}(1-\Phi(k, \omega, x)) .
$$

For $k>0, k^{2} \notin \sigma_{p}(H), \omega \in S^{2}, x \in \mathbb{R}^{3}$

$$
\Psi_{+}(k, \omega, x)=\Psi(-k,-\omega, x), \quad \Psi_{-}(k, \omega, x)=\Psi(k, \omega, x) .
$$

\section{Analytic Continuation}

Under our assumptions on $V$ the scattering operator $S$ exists and is unitary on $L_{2}$. The generalized Fourier transforms $F_{ \pm}$are partial isometries with initial space 
$\mathscr{H}_{\text {ac }}\left(H_{1}\right)$ and final space $L_{2}$, defined for $f \in C_{0}\left(\mathbb{R}^{3}\right), k>0, \omega \in S^{2}$ by

$$
\left(F_{ \pm} f\right)(k, \omega)=(2 \pi)^{3 / 2} \int_{\mathbb{R}^{3}} f(x) \bar{\Psi}_{ \pm}(k, \omega, x) d x .
$$

Letting $\mathscr{F}$ denote the Fourier-Plancherel transform and setting $\hat{S}=\mathscr{F} S \mathscr{F}-1$, we have $\hat{S} F_{-}=F_{+}$, hence for $f \in C_{0}\left(\mathbb{R}^{3}\right)$,

$$
\widehat{S}\left[\int_{\mathbb{R}^{3}} f(x) \bar{\Psi}_{-}(k, \omega, x) d x\right]=\int_{\mathbb{R}^{3}} f(x) \bar{\Psi}_{+}(k, \omega, x) d x .
$$

Moreover, $S H_{0}=H_{0} S$, so $\hat{S}$ is diagonalized in momentum representation, $\widehat{S}=\int_{0}^{\infty} \oplus S(k) d k$, where for $k>0, f \in C_{0}\left(\mathbb{R}^{3}\right)$,

$$
S(k) \int_{\mathbb{R}^{3}} f(x) \bar{\Psi}_{-}(k, \cdot, x) d x=\int_{\mathbb{R}^{3}} f(x) \bar{\Psi}_{+}(k, \cdot, x) d x .
$$

Since $\bar{\Psi}_{ \pm}(k, \cdot, \cdot) \in C\left(\mathbb{R}^{3}, h\right)$ and $S(k) \quad$ is unitary on $h$, also $S(k) \bar{\Psi}_{-}(k, \cdot, \cdot) \in C\left(\mathbb{R}^{3}, h\right)$. Hence, $(2.1)$ for all $f \in C_{0}\left(\mathbb{R}^{3}\right)$ implies for $k>0, x \in \mathbb{R}^{3}$,

$$
S(k) \bar{\Psi}_{-}(k, \cdot, x)=\bar{\Psi}_{+}(k, \cdot, x) .
$$

Using (1.1), the above result (2.2) may be expressed in terms of $\Psi(k, \omega, x)$ as follows.

Lemma 2.1. The scattering matrix $S(k)$ satisfies $S(k) \Psi(-k, \cdot, x)=\Psi(k,-\cdot, x)$, $x \in \mathbb{R}^{3}, k>0$.

Definition. Let $v$ be a real-valued, measurable function satisfying (i) and (ii). Let $\mathcal{O} \subset \mathbb{C}^{-}$be a domain with $\partial \mathcal{O} \cap \mathbb{R}^{+}=I$. The operator $V$ of multiplication by $v$ is called $\mathcal{O}$-analytic, if the scattering matrix $S(k)$ has a continuous extension $\widetilde{S}(k)$ from $I$ to $\mathcal{O} \cup I$, such that $\tilde{S}(k)$ is analytic in $\mathcal{O}$. For any set $S \subset \mathbb{C}$ we let $S_{b}$ $=\{k \in S|| \operatorname{Im} k \mid<b\}$.

Theorem 2.2. Assume that $V$ is $\mathcal{O}$-analytic.

1) For fixed $x \in \mathbb{R}^{3}$, the function $\Psi(k, \cdot, x)$ has an $\mathfrak{H}$-valued meromorphic continuation $\widetilde{\Psi}(k, \cdot, x)$ from $\mathbb{C}^{+}$to $\mathcal{O} \cup I$ with poles contained in $\Sigma \cup \bar{\Sigma}$, defined for $k \in(\mathcal{O} \cup I) \backslash \bar{\Sigma}, x \in \mathbb{R}^{3}, b y$

$$
\widetilde{\Psi}(k, \cdot, x)=R \widetilde{S}(k) \Psi(-k, \cdot, x)
$$

where $R$ is the reflection operator in $\mathfrak{H},(R \sigma)(\omega)=\sigma(-\omega)$.

2) $\widetilde{\Psi}(k, \cdot, \cdot) \in \mathscr{H}_{2, \mathfrak{H}}^{-b}$ for $|\operatorname{Im} k|<b$, and $\widetilde{\Psi}(k, \cdot, \cdot)$ is a meromorphic, $\mathscr{H}_{2 . \mathfrak{H}}^{-b}$-valued function of $k$ in $\left(\mathbb{C}^{+} \cup I \cup \mathcal{O}\right)_{b}$ with poles contained in $\Sigma \cup \bar{\Sigma}$.

Proof. 1) Define $\widetilde{\Psi}(k, \cdot, x)$ for $x \in \mathbb{R}^{3}, k \in(\mathcal{O} \cup I) \backslash \bar{\Sigma}$ by (2.3). For $k \in I \backslash \bar{\Sigma}$, by Lemma 2.1,

$$
\widetilde{\Psi}(k, \cdot, x)=\Psi(k, \cdot, x) .
$$

By Theorem 1.5 and Definition 1.6, $\widetilde{\Psi}(k, \cdot, x)$ is continuous on $(\mathcal{O} \cup I) \backslash \bar{\Sigma}$ and meromorphic in $\mathcal{O}$ with poles contained in $\bar{\Sigma}_{i}$. Using again Theorem 1.5, we conclude that for $x \in \mathbb{R}^{3}, \tilde{\Psi}(k, \cdot, x)$ is meromorphic in $\mathbb{C}^{+} \cup I^{\prime} \cup \mathcal{O}$, where $I^{\prime}$ $=I \backslash\left\{\alpha \mid \alpha^{2} \in \sigma_{p}(H)\right\}$, with poles at most at $\Sigma_{i} \cup \bar{\Sigma}_{i}$. The fact that $\left\{\alpha \mid \alpha^{2} \in \sigma_{p}(H)\right\}$ are 
(simple) poles of $\widetilde{\Psi}(k, \cdot, x)$ can be proved using the existence of $\lim _{z \rightarrow \alpha}(z-\alpha) R_{1}(z)$. This concludes the proof of 1).

2) By Theorem 1.5 , for $|\alpha| \leqq 2$,

$$
\int_{\mathbb{R}^{3}}\left\|D^{\alpha} \widetilde{\Psi}(k, \cdot, x)\right\|_{\mathfrak{H}}^{2} e^{-2 b|x|} d x \leqq\|\widetilde{S}(k)\|^{2} \int_{\mathbb{R}^{3}}\left\|D^{\alpha} \Psi(-k, \cdot, x)\right\|_{\mathfrak{H}}^{2} d x .
$$

The analyticity properties of $\widetilde{\Psi}(k, \cdot, \cdot)$ as an $\mathscr{H}_{2, \mathfrak{h}}^{-b}$-valued function follows from local boundedness and weak analyticity on a dense set of $D^{\alpha} \widetilde{\Psi}(k, \cdot, \cdot)$ for $|\alpha| \leqq 2$, and 2) is proved.

Lemma 2.3. Assume that $V$ is $\mathcal{O}$-analytic. The trace operator $T(k)$ defined for $k>0$ by

$$
T(k) f=\left(F_{+} f\right)(k, \cdot)
$$

has a $\mathscr{B}\left(\mathscr{H}^{b}, \mathfrak{H}\right)$-valued, meromorphic extension $\widetilde{T}(k)$ from $I$ to $\left(\mathbb{C}^{+} \cup I \cup \mathcal{O}\right)_{b}$ with poles at most at $\Sigma \cup \bar{\Sigma}$, given by

$$
\widetilde{T}(k) f=(2 \pi)^{-3 / 2} R \int_{\mathbb{R}^{3}} f(x) \widetilde{\Psi}(k, \cdot, x) d x .
$$

Moreover, $\widetilde{T}^{*}(\bar{k})$, given by

$$
\left(\widetilde{T}^{*}(\bar{k}) \sigma\right)(x)=(2 \pi)^{-3 / 2} \int_{S^{2}} \sigma(\omega) \widetilde{\Psi}(\bar{k},-\omega, x) d \omega
$$

is in $\mathscr{B}\left(\mathfrak{H}, \mathscr{H}_{2, \mathfrak{H}}^{-b}\right)$.

Proof. It follows from Theorem 2.2,2) that for $f \in \mathscr{H}^{b}, \widetilde{T}(k) f$ as given by (2.4) is a meromorphic, $\mathfrak{H}$-valued function of $k \in\left(\mathbb{C}^{+} \cup I \cup \mathcal{O}\right)_{b}$ with poles contained in $\Sigma \cup \bar{\Sigma}$. From the boundedness of $\widetilde{\Psi}(k, \cdot, \cdot) \|_{\mathscr{H}_{\mathfrak{H}^{b}}}$ on compact sets follows that $\|\widetilde{T}(k)\|_{\mathscr{B}(\mathscr{H} b, \mathfrak{S})}$ is locally bounded and hence $\widetilde{T}(k)$ is a meromorphic $\mathscr{B}\left(\mathscr{H}^{b}, \mathfrak{H}\right)$-valued function on $\left(\mathbb{C}^{+} \cup I \cup \mathcal{O}\right)_{b}$. A simple calculation yields (2.5), and it follows from Theorem 1.5 that $\widetilde{T}^{*}(\bar{k}) \in \mathscr{B}\left(\mathfrak{H}, \mathscr{H}_{2, \mathfrak{H}}^{-b}\right)$. The lemma is proved.

Theorem 2.4. Assume that $V$ is $\mathcal{O}$-analytic. The resolvent $R_{1}(k)$ has a meromorphic, $\mathscr{B}\left(\mathscr{H}^{b}, \mathscr{H}_{2}^{-b}\right)$-valued continuation $\widetilde{R}_{1}(k)$ from $\mathbb{C}^{+}$to $I \cup \mathcal{O}_{b}$ with poles contained in $\bar{\Sigma}$.

Proof. We use the well-known identity, valid for $k>0, k^{2} \notin \sigma_{p}(H)$,

$$
R_{1+}(k)=R_{1+}(-k)+\pi i k T^{*}(k) T(k) .
$$

Using Lemma 2.3, we define the $\mathscr{B}\left(\mathscr{H}^{b}, \mathscr{H}_{2}^{-b}\right)$-valued function $\widetilde{R}_{1}(k)$ for $k \in \mathcal{O}_{b} \backslash \bar{\Sigma}$ by

$$
\widetilde{R}_{1}(k)=R_{1}(-k)+\pi i k \widetilde{T}^{*}(\bar{k}) \widetilde{T}(k) .
$$

Setting $\widetilde{R}_{1}(k)=R_{1+}(k)$ for $k \in I \backslash \Sigma$, we obtain the theorem from (2.6) and Lemma 2.3.

Theorem 2.5. Assume that $V$ is $\mathcal{O}$-analytic. Let $W$ be a symmetric operator of the form

$$
W=e^{-b|x|} Q e^{-b|x|}, Q \in \mathscr{C}\left(H_{2}\left(\mathbb{R}^{3}\right), L_{2}\left(\mathbb{R}^{3}\right)\right) .
$$

Let $\mathrm{H}_{2}$ be the selfadjoint operator on $\mathscr{D}_{\mathrm{H}_{2}}=\mathscr{D}_{\mathrm{H}_{1}}=\mathscr{D}_{\mathrm{H}_{0}}$ defined by

$$
H_{2}=H_{1}+W=H_{0}+V+W,
$$


and

$$
R_{2}(k)=\left(H_{2}-k^{2}\right)^{-1} \quad \text { for } \quad k^{2} \in \varrho\left(H_{2}\right) .
$$

Let $S_{12}(k)$ be the scattering matrix of the pair $\left(H_{1}, H_{2}\right)$ associated with the spectral representation of $H_{1, \text { ac }}$ defined by $T(k)$ (cf. [8]).

1) $R_{2}(k)$ has a $\mathscr{B}\left(\mathscr{H}^{b}, \mathscr{H}_{2}^{-b}\right)$-valued, meromorphic continuation $\widetilde{R}_{2}(k)$ from $\mathbb{C}^{+}$to $\left(I \cup \mathcal{O}_{b}\right) \backslash \bar{\Sigma}$, given by

$$
\widetilde{R}_{2}(k)=\widetilde{R}_{1}(k)\left(1+W \widetilde{R}_{1}(k)\right)^{-1} .
$$

2) $S_{12}(k)$ has a meromorphic extension $\widetilde{S}_{12}(k)$ from I to $\mathcal{O}_{b} \backslash \bar{\Sigma}_{i}$ with the same poles as $\widetilde{R}_{2}(k)$, given by

$$
\widetilde{S}_{12}(k)=1-\pi i k \widetilde{T}(k)\left(W-W \widetilde{R}_{2}(k) W\right) \widetilde{T}^{*}(\bar{k}) .
$$

Proof. 1) By Theorem 2.4, $W R_{1}(k)$ has a $\mathscr{C}\left(\mathscr{H}^{b}\right)$-valued analytic continuation $W \widetilde{R}_{1}(k)$ from $\mathbb{C}^{+}$to $\left(I \cup \mathcal{O}_{b}\right) \backslash \bar{\Sigma}$. By the analytic Fredholm theorem, $\left(1-W \widetilde{R}_{1}(k)\right)^{-1}$ is meromorphic in $\left(I \cup \mathcal{O}_{b}\right) \backslash \bar{\Sigma}$. Using the $2^{\text {nd }}$ resolvent equation, we obtain 1$)$.

2) Using the representation of $H_{1 \text {, ac }}$ as $k^{2}$ on $L_{2}\left(\mathbb{R}^{+}, \mathfrak{H} ; k^{2} d k\right)$ defined by $T(k)$, the scattering matrix $S_{12}(k)$ is given for $k>0$ (cf. [8]) by

$$
S_{12}(k)=1-\pi i k T(k)\left(W-W R_{2+}(k) W\right) T^{*}(k) .
$$

It follows from $(2.10), 1)$ and Lemma 2.3, that $S_{12}(k)$ has a meromorphic extension $\widetilde{S}_{12}(k)$ to $\mathcal{O}_{b} \backslash \bar{\Sigma}$ with poles at most at the poles of $\widetilde{R}_{2}(k)$. The fact that the poles of $\widetilde{S}_{12}(k)$ and $\widetilde{R}_{2}(k)$ coincide follows from the next lemma.

Lemma 2.6. For $k \in \mathcal{O}_{b} \backslash \bar{\Sigma}_{i}, \mathcal{N}\left(\tilde{S}_{12}^{-1}(k)\right)$ and $\mathscr{N}\left(1+W \widetilde{R}_{1}(k)\right)$ are isomorphic via the maps

$$
\mathscr{N}\left(1+W \tilde{R}_{1}(k)\right) \ni \Omega \rightarrow \sigma=\widetilde{T}(k) \Omega \in \mathscr{N}\left(\tilde{S}_{12}^{-1}(k)\right)
$$

with the inverse $Z(k)$ defined by

$$
\Omega=Z(k) \sigma=-\pi i k\left(1-W R_{2}(-k)\right) W \widetilde{T}^{*}(\bar{k}) \sigma .
$$

Proof. 1) Let $\Omega \in \mathscr{N}\left(1+W \tilde{R}_{1}(k)\right.$. Then $\sigma \neq 0$, since otherwise by (2.7) $\Omega \in \mathscr{N}\left(1+W R_{1}(-k)\right)$ implying $R_{1}(-k) \Omega \in \mathscr{N}\left(H_{2}-k^{2}\right)$, a contradiction. Using the expression for $\widetilde{S}_{12}^{-1}$ obtained from (2.8) on replacing $i$ by $-i$ and $\widetilde{R}_{2}(k)$ by $R_{2}(-k)$, we get in view of (2.7),

$$
\begin{aligned}
\tilde{S}_{12}^{-1}(k) \sigma= & \left(1+\pi i k \widetilde{T}(k)\left(1-W R_{2}(-k)\right) W \widetilde{T}^{*}(\bar{k}) \widetilde{T}(k) \Omega\right. \\
= & \widetilde{T}(k) \Omega+\widetilde{T}(k)\left(1-W R_{2}(-k)\right) \\
& \times\left[\left(1+W \widetilde{R}_{1}(k)\right)-\left(1+W R_{1}(-k)\right)\right] \Omega=0 .
\end{aligned}
$$

2) Assume that $\sigma \in \mathcal{N}\left(\tilde{S}_{12}^{-1}(k)\right)$, i.e.,

$$
\sigma-\widetilde{T}(k) Z(k) \sigma=0 .
$$

Applying $Z(k)$, setting $\Omega=Z(k) \sigma$ and using (2.7), we get

$$
\begin{aligned}
\Omega-Z(k) \widetilde{T}(k) \Omega & =\Omega+\left(1-W R_{2}(-k)\right) W\left[\widetilde{R}_{1}(k)-R_{1}(-k)\right] \Omega \\
& =\left(1+W \widetilde{R}_{1}(k)\right) \Omega=0 .
\end{aligned}
$$

The lemma follows from 1) and 2). 
We finally investigate the analyticity properties of the scattering matrix $S_{2}(k)$ of the pair $\left(\mathrm{H}_{0}, \mathrm{H}_{2}\right)$.

Theorem 2.7. Under the assumptions of Theorem 2.5 the scattering matrix $S_{2}(k)$ has a meromorphic extension $\tilde{S}_{2}(k)$ from $I$ to $\mathcal{O}_{b} \backslash \bar{\Sigma}_{i}$ with poles at most at the poles of $\widetilde{R}_{2}(k)$.

Proof. This follows from Theorem 2.5 and the following identity, valid for $k>0$,

$$
S_{12}(k) S_{1}(k)=S_{2}(k),
$$

which we shall now establish.

Using for $H_{1, \text { ac }}$ the representation as $k^{2}$ on $L_{2}\left(\mathbb{R}^{+}, \mathfrak{H} ; k^{2} d k\right)$ defined by $T(k)$, the generalized Fourier transforms $F_{12 \pm}$ of the pair $\left(H_{1, \mathrm{ac}}, H_{2, \mathrm{ac}}\right)$ are given (cf. [8]) for $f \in L_{2, s}$ and $k>0, k^{2} \notin \sigma_{p}\left(H_{1} \cup \sigma_{p}\left(H_{2}\right)\right)$, by

$$
\begin{aligned}
\left(F_{12+} f\right)(k) & =T(k)\left(1-W R_{2+}(k)\right) f=T_{0}(k)\left(1-V R_{1+}(k)\right)\left(1-W R_{2+}(k)\right) f \\
& =T_{0}(k)\left(1-(V+W) R_{2+}(k)\right) f=\left(F_{2+} f\right)(k),
\end{aligned}
$$

where

$$
T_{0}(k) f=(\mathscr{F} f)(k, \cdot),
$$

and

$$
\begin{aligned}
\left(F_{12-} f\right)(k) & =T(k)\left(1-W R_{2+}(-k)\right) f=\left(F_{1+}\left(1-W R_{2+}(-k)\right) f\right)(k) \\
& =S_{1}(k)\left(F_{1-}\left(1-W R_{2+}(-k)\right) f\right)(k) \\
& =S_{1}(k) T_{0}(k)\left(1-V R_{1+}(-k)\right)\left(1-W R_{2+}(-k)\right) f \\
& =S_{1}(k) T_{0}(k)\left(1-(V+W) R_{2+}(-k)\right) f=S_{1}(k)\left(F_{2-} f\right)(k) .
\end{aligned}
$$

By (2.12) and (2.13), for $f \in L_{2, s}, k>0, k^{2} \notin \sigma_{p}\left(H_{1}\right) \cup \sigma_{p}\left(H_{2}\right)$,

$$
\begin{aligned}
S_{2}(k)\left(F_{2-} f\right)(k) & =\left(F_{2+} f\right)(k)=\left(F_{12+} f\right)(k) \\
& =S_{12}(k)\left(F_{12-} f\right)(k)=S_{12}(k) S_{1}(k)\left(F_{2-} f\right)(k) .
\end{aligned}
$$

From (2.14) follows (2.11) for $k>0, k^{2} \notin \sigma_{p}\left(H_{1}\right) \cup \sigma_{p}\left(H_{2}\right)$. Since $S_{12}(k), S_{1}(k)$, and $S_{2}(k)$ are continuous on $\mathbb{R}^{+}$(cf. [7]) and $\sigma_{p}\left(H_{1}\right)$ and $\sigma_{p}\left(H_{2}\right)$ are discrete sets in $\mathbb{R}^{+}$, this implies (2.11) for all $k>0$, and the proof is complete.

\section{Appendix 1}

Lemma A 1. Let $f$ and $g$ be functions in $L_{2}$ with compact support, and let $\alpha_{0} \in \mathbb{R} \backslash\{0\}$. Then there exists $0<\delta_{0}<\left|\alpha_{0}\right|$ such that the following limits exist, uniformly in $\left\{k=\alpha+i \beta|| \alpha-\alpha_{0} \mid \leqq \delta_{0}, 0 \leqq \beta \leqq \delta_{0}\right\}$,

$$
\lim _{\varepsilon \downarrow 0}\left(f, R_{0}(\beta, k+i \varepsilon) g\right) .
$$

Proof. Taking Fourier transforms, we have

$$
I=\left(f, R_{0}(\beta, k+i \varepsilon) g\right)=\int_{\mathbb{R}^{3}} \hat{f}(\xi) \overline{\hat{g}}(\xi)\left(\xi^{2}-\alpha^{2}+2 \varepsilon \beta+\varepsilon^{2}+2 i\left(\beta \xi_{1}-\beta \alpha-\varepsilon \alpha\right)\right)^{-1} d \xi .
$$


Since $\hat{f}(\xi)$ and $\bar{g}(\bar{\xi})$ are entire analytic functions of $\xi_{1}, \xi_{2}, \xi_{3}$, we can deform the manifold of integration in $\mathbb{C}^{3}$ as follows.

Let $\alpha_{0}>0\left(\alpha_{0}<0\right.$ is analogous). It suffices to consider $\beta=0$.

We write the integral in spherical coordinates as follows.

$$
I=I(\varepsilon)=\int_{S^{2}} \sin \theta d \theta d \varphi \int_{\mathbb{R}^{+}} d r \hat{f}(r, \theta, \varphi) \overline{\hat{g}}(r, \theta, \varphi)\left(r^{2}-\alpha^{2}+\varepsilon^{2}-2 i \varepsilon \alpha\right)^{-1} .
$$

Deforming the radial integration path $\mathbb{R}^{+}$into the curve $\Gamma$ indicated on Fig. 1, where $0<\delta<\alpha$, we get

$$
\begin{aligned}
I(\varepsilon)= & \int_{S^{2}} \sin \theta d \theta d \varphi \int_{\Gamma} d z \hat{f}(z, \theta, \varphi) \overline{\hat{g}}(\bar{z}, \theta, \varphi) \\
& \times\left(z^{2}-\alpha^{2}+2 \varepsilon \beta+\varepsilon^{2}+2 i(\beta z \sin \theta \cos \varphi-\beta \alpha-\varepsilon \alpha)^{-1},\right.
\end{aligned}
$$

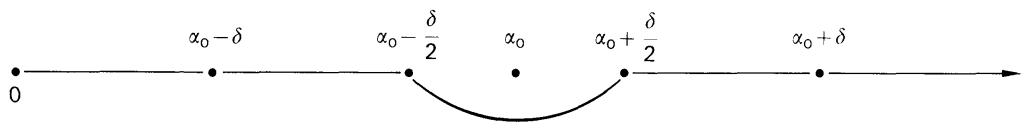
$\underset{\leqq \delta / 4}{\text { Fig. 1. This yields } I(\varepsilon) \underset{\varepsilon \downarrow 0}{\longrightarrow} \int_{S^{2}} \sin \theta d \theta d \varphi \int_{\Gamma} d z \hat{f}(z, \theta, \varphi) \bar{g}(\bar{z}, 0, \varphi)\left(z^{2}-\alpha^{2}\right)^{-1} \text {, uniformly for }\left|\alpha-\alpha_{0}\right|}$

\section{Appendix 2}

Lemma A 2.1. Let $b_{0}>0$ be fixed. For $|\beta|<\beta_{0}$ and $\mathbb{C}^{b_{0}}=\left\{a+i b \mid b>b_{0}\right\}$, we have

$$
\sigma_{d}(H(i \beta)) \cap\left\{z^{2} \mid z \in \mathbb{C}^{b_{0}}\right\}=\left\{-b^{2} \in \sigma_{d}(H) \mid b>b_{0}\right\} .
$$

Proof. We set

$$
H(z)=e^{-i z} H e^{i z}=-\Delta-2 i z \frac{\partial}{\partial x_{1}}+z^{2}+V .
$$

For $\beta$ fixed

$$
H(\alpha+i \beta)=e^{-i \alpha x_{1}} H(i \beta) e^{i \alpha x_{1}} .
$$

The operators $H(z)$ form an entire self-adjoint, analytic family of operators of type A. For fixed $\beta$ the operators $H(\alpha+i \beta)$ are unitarily equivalent. The essential spectrum $\sigma_{e}(z)$ of $H(z)$ is the parabolic region $\left\{\zeta^{2}|\operatorname{Im} \zeta| \leqq|\beta|\right\}$ (for $\beta=0$ coinciding with $\overline{\mathbb{R}^{+}}$). Thus, $\mathbb{C}^{b_{0}} \cap \sigma_{e}(z)=\emptyset$ for $|\beta|<b_{0}$. A discrete eigenvalue $\lambda$ of $H\left(\alpha^{\prime}+i \beta^{\prime}\right)$, $\left|\beta^{\prime}\right|<b_{0}$, remains a discrete eigenvalue of $H\left(\alpha+i \beta^{\prime}\right)$ for all $\alpha \in \mathbb{R}$ and hence, by analyticity of $H(z)$, for all $\alpha \in \mathbb{R},|\beta|<b_{0}$.

The lemma follows.

Lemma A 2.2. Let $\beta>0$ be fixed and let $k=a+i b, a \in \mathbb{R}, b>\beta$. Then the equation

$$
\phi+R_{0}(\beta, k) V \phi=0
$$

has a solution $\phi \in H_{2,-s}, \phi \neq 0$, if and only if $a=0$ and $k^{2}=-b^{2} \in \sigma_{d}(H(\beta))$ with

$$
\left(H(i \beta)+b^{2}\right) \phi=0 \text {. }
$$


Proof. 1) If $\phi \in H_{2}$ satisfies (2), then applying $R_{0}(\beta, i b) \in \mathscr{B}\left(L_{2}, H_{2}\right)$ to (2), we get, since $V \phi \in L_{2},(1)$ where $\phi \in H_{2,-s}$.

2) Let $\phi \in H_{2,-s}$ and assume (1). Then $V \phi \in L_{2, s} \subset L_{2}$ and hence $\phi=-R_{0}(\beta, k) V \phi \in H_{2}$. Applying $H_{0}(\beta)-k^{2}$ to (1), we get (2) and hence by Lemma A2.1, $a=0$ and $k^{2}=-b^{2} \in \sigma_{d}(H(i \beta)$.

Lemma A 2.3. For $\beta>0, k=\alpha+i \beta$,

$$
R_{0+}(\beta, k)=\lim _{\beta^{\prime} \uparrow \beta}\left(-\Delta-2 \beta^{\prime} \frac{\partial}{\partial x_{1}}-\beta^{\prime 2}-k^{2}\right)^{-1} .
$$

Proof. This follows from the fact, proved in Theorem 1.3, that

$$
\left\|R_{0}(\beta, k+i \varepsilon)-R_{0+}(\beta, k)\right\| \mathrm{B}\left(L_{2, s}^{1}, H_{2,-s}\right) \underset{\varepsilon \rightarrow 0}{\longrightarrow} 0,
$$

uniformly on compact sets, together with the norm-continuity of $R_{0+}(\beta, k)$.

\section{Lemma A 2.4.}

$$
\left\{k=\alpha+i \beta \in \mathbb{C}^{+} \mid \mathscr{N}\left(1+R_{0+}\left(\beta, z^{2}\right) V\right) \neq\{0\}\right\}=\left\{i \beta \mid-\beta^{2} \in \sigma_{d}(H)\right\} .
$$

Proof. Fix $k=\alpha+i \beta, \alpha \in \mathbb{R}, \beta>0$. By Lemma A2.1, for $0 \leqq \beta^{\prime}<\beta$,

$$
\sigma_{d}\left(H\left(i \beta^{\prime}\right)\right) \cap\left\{k^{2} \mid \operatorname{Im} k \geqq \beta\right\}=\left\{-b^{2} \in \sigma_{d}(H) \mid b \geqq \beta\right\} .
$$

Hence, by Lemma A 2.2, there exists a circle $C$ with center -1 , separating - 1 from the rest of the spectrum of the operator $R_{0}\left(\beta^{\prime}, k\right) V \in \mathscr{C}\left(H_{2,-s}\right)$ for $\alpha \in \mathbb{R}$, $0 \leqq \beta^{\prime}<\beta$.

Let

$$
P\left(\beta^{\prime}, k\right)=-\frac{1}{2 \pi i} \int_{C}\left(-\lambda+R_{0}\left(\beta^{\prime}, k\right) V\right)^{-1} d \lambda
$$

By Lemma A2.3

$$
\lim _{\beta^{\prime} \uparrow \beta} R_{0}\left(\beta^{\prime}, k\right) V=R_{0+}(\beta, k) V
$$

in the uniform operator topology of $\mathscr{B}\left(H_{2,-s}\right)$.

It follows that

$$
\left(-\lambda+R_{0}\left(\beta^{\prime}, k\right) V\right)^{-1} \underset{\beta^{\prime} \uparrow \beta}{\longrightarrow}\left(-\lambda+R_{0}(\beta, k) V\right)^{-1}
$$

in the uniform operator topology of $\mathscr{B}\left(H_{2,-s}\right)$, uniformly for $\lambda \in C$. Hence, in the same topology

$$
P\left(\beta^{\prime}, k\right) \underset{\beta^{\prime} \uparrow \beta}{\longrightarrow}-\frac{i}{2 \pi i} \int_{C}\left(-\lambda+R_{0+}(\beta, k) V\right)^{-1} d \lambda=P_{+}(\beta, k),
$$

where $P_{+}(\beta, k)$ is a projection on the algebraic null space of $1+R_{0+}(\beta, k) V$. 
It follows that $P_{+}(\beta, k) \neq 0$ if and only if $P\left(\beta^{\prime}, k\right) \neq 0$ for all $\beta^{\prime}<\beta$. By Lemma A2.1 this holds if and only if $\alpha=0$ and $-\beta^{2} \in \sigma_{d}(H)$, and the lemma is proved.

Acknowledgement. I want to thank Ira Herbst for a valuable discussion, suggesting the possibility of proving the estimates of Sect. 1 for fixed $\omega \in S^{2}$. I also thank Erik Skibsted for pointing out an error in the original proof of Lemma A 2.3 and indicating the proof given.

\section{References}

1. Agmon, S.: Spectral properties of Schrödinger operators and scattering theory. Ann. Sc. Norm. Super. Pisa, Ser. IV, II, 151-218 (1975)

2. Agmon, S.: Spectral theory of Schrödinger operators on euclidean and non-euclidean spaces. Commun. Pure Appl. Math. XXXIX, 3-16 (1986)

3. Babbitt, D., Balslev, E.: Local distortion techniques and unitarity of the $S$-matrix for the 2-body problem. J. Math. Anal. Appl. 54, 316-347 (1976)

4. Balslev, E.: Analytic scattering theory of two-body Schrödinger operators. J. Funct. Anal. 29, 375-396 (1978)

5. Balslev, E.: Local spectral deformation techniques for Schrödinger operators. J. Funct. Anal. 58, 79-105 (1984)

6. Balslev, E.: Resonance functions for radial Schrödinger operators. J. Math. Anal. Appl. 123, 339-365 (1987)

7. Jensen, A.: Resonances in an abstract analytic scattering theory. Ann. Inst. H. Poincaré Phys. Théor. XXXIII, 209-223 (1980)

8. Kuroda, S.T.: Scattering theory for differential operators. I. J. Math. Soc. Jpn. 25, 75-104 (1973)

9. Sigal, I.M.: Complex transformation methods and resonances in one-body quantum systems. Ann. Inst. H. Poincaré Phys. Théor. 41, 103-114 (1984); 41, 333 (1984)

10. Saitō, Y.: Extended limiting absorption method and analyticity of the $S$-matrix. J. Rcine Angew. Math. 343, 1-22 (1983)

11. Balslev, E., Skibsted, E.: Resonance theory for two-body Schrödinger operators (to appear)

Communicated by B. Simon

Received June 16, 1987 